\title{
$I-V$ Characteristics of Resistive Oxides: DC versus Pulsed Measurements
}

\author{
B. Fisher, J. Genossar, K.B. Chashka, L. Patlagan \\ AND G.M. REISNER \\ Physics Department, Technion, Haifa 32000, Israel
}

\begin{abstract}
We investigated pulsed and DC $I-V$ characteristics of a variety of resistive oxides. Examples of pulsed, compared with DC characteristics, are shown for samples of $\mathrm{La}_{1.2} \mathrm{Sr}_{1.8} \mathrm{Mn}_{2} \mathrm{O}_{7}$ (a double layer manganite), $\mathrm{Pr}_{2 / 3} \mathrm{Ca}_{1 / 3} \mathrm{MnO}_{3}$ and $\mathrm{Bi}_{1 / 2} \mathrm{Sr}_{1 / 2} \mathrm{MnO}_{3}$ (charge-ordered manganites), $\mathrm{Sr}_{2} \mathrm{FeMoO}_{6}, \mathrm{Sr}_{2} \mathrm{CrWO}_{6}$ and $\mathrm{Ba}_{2} \mathrm{MnReO}_{6}$ (double perovskites). For pulsed measurements, single pulses in the ms range were applied. For short-rise-time square pulses, the Joule heating is negligible, as long as the response remains independent of time. The DC $I-V$ characteristics were measured up to current runaway in the negative resistance regime; $\Delta T$ never exceeded a fraction of a degree. In most cases the DC characteristics mask a perfectly ohmic or moderately non-ohmic conductivity obtained by pulsed measurements. This demonstrates that the widely used DC I-V measurements in the high current regime are frequently misleading.
\end{abstract}

PACS numbers: 72.20.Ht, 75.47.Lx, 77.80.Fm

\section{Introduction}

Electronic effects giving rise to non-ohmic conductivity under high electric fields have been popular topics of investigations over many years. Field-enhanced tunneling or hopping and many other bulk or interface effects frequently compete with thermal or electro-thermal effects when investigated using direct current (DC).

The thermal theory of non-ohmic conductivity, negative differential resistance and breakdown is very old, with a rich literature going back over a century [1]. Nevertheless, many published $I-V$ characteristics and in particular the most recent ones, aimed at the investigation of electronic effects, were obtained using DC. Many reports on phenomena observed under high DC currents have been published in important journals and some dramatic results became quite famous. An early case is the report on reversible electrical switching phenomena in disordered structures [2] which gave rise to a number of controversial interpretations, put forward by some of the prominent solid state physicists at the time [3]. Even- 
tually the role of the Joule heating in the switching process was demonstrated when thermal filaments were visually observed [4]. Charge ordering (CO), often associated with spin (antiferromagnetic (AF)) ordering [5] and its collapse in manganite perovskites [6], have been the subject of intense investigations over the past decade. An important role in these investigations was played by the study of nonlinear $I-V$ characteristics of various charge-ordered manganites. The first such observation was the dramatic decrease in the resistance of a single crystal of $\mathrm{Pr}_{0.7} \mathrm{Ca}_{0.3} \mathrm{MnO}_{3}$, at temperatures below the $\mathrm{CO}$ transition temperature $\left(T_{\mathrm{CO}}\right)[7]$, induced by electric fields of the order of $10^{3} \mathrm{~V} / \mathrm{cm}$; it was proposed that the current switching may involve dielectric breakdown. This initial experiment was followed by extensive studies of nonlinear conductivity of single crystals, films and polycrystalline samples of different CO manganite compounds, carried out in many labs. Various interpretations were proposed including (partial) melting of the CO, or depinning of charge density waves related to CO. (For a short review see Ref. [8].) The $I-V$ characteristics were obtained using DC currents; the Joule heating was usually ruled out by calculating the expected temperature increment $\Delta T$ and/or by monitoring it close to the samples' surface. However, both the calculations and the measurements showing $\Delta T$ to be negligible were misleading, being based on the assumption that the current flow throughout the cross-section of the sample, and thus its temperature, are homogeneous. A recent elaborate investigation of polycrystalline samples of $\operatorname{Pr}_{0.8} \mathrm{Ca}_{0.2} \mathrm{MnO}_{3}$ (with no $\mathrm{CO}$ but exhibiting nonlinear conductivity) has shown that the internal thermal gradient caused by the Joule heating was at the origin of the $I(V)$ nonlinearity [8]. In this experiment the crystal unit cell was used as an internal thermometer employing X-ray and neutron diffractions. The role of the Joule heating in electronic transport measurements of manganites is gradually percolating into this field as a possible interpretation of some of the experimental results [9]. An old technique for preventing Joule heating errors in high electric field measurements and confirming its absence, consists of applying pulsed currents and following the time dependence of the response on an oscilloscope. For short-rise-time square pulses, the Joule heating is negligible, as long as the response remains independent of time.

In the course of our studies of various transition metal oxides, manganites without [10] and with [11] CO and double perovskites [11, 12], we have routinely measured both pulsed and DC $I-V$ characteristics of various samples at different temperatures. The motivation for the study of nonlinear conductivity of those systems was the need to understand the mechanisms of transport across grain boundaries or other weak links. In this report we emphasize the importance of employing pulsed currents in all nonlinear conductivity measurements. The experimental methods are summarized in Sect. 2. Pulsed characteristics compared with DC $I-V$ characteristics are shown in Sect. 3. The comparison shows that DC characteristics mask a perfectly ohmic or moderately non-ohmic conductivity obtained by pulsed measurements. 


\section{Experimental}

The ohmic resistivities $(\rho)$ of the investigated samples had negative temperature coefficients; for fixed temperatures, $\rho$ of different samples ranged over many orders of magnitude (up to $\approx 10^{6} \Omega \mathrm{cm}$ ). All our samples were bars of rectangular cross-section. The quality of the current contacts and of the voltage probes and the small size of the latter are of crucial importance and were given special care. The experimental setup for the four-probe, pulsed and DC $I-V$ measurements is described in Ref. [11]. For the pulsed measurements, single pulses in the millisecond range were applied from a Keithley 237 high voltage source. The maximal voltage was $150 \mathrm{~V}$ for currents up to $10 \mathrm{~mA}$. Pairs of voltage drops were recorded simultaneously using two channels of a Tektronix 2221A digital storage oscilloscope. Beyond the finite rise time of the pulses, which depends on the sample resistance, the voltage drops, as a function of time, were constant. The effect of heating could be observed for long pulses. In only a few cases the measurements had to be interrupted when the pulse rise time and the heating overlapped.

DC $I-V$ characteristics were measured over several orders of magnitude. Below current runaway, in the negative resistance range, the measured surface $\Delta T$ never exceeded a fraction of a degree.

\section{Experimental results and discussion}

Figures 1-4 present plots of current density $(J)$ versus electric field $(E)$ for polycrystalline samples of different systems, at various temperatures. $J$ and $E$ were obtained from the total current $I$ and the voltage drop between the voltage probes $\Delta V$, divided by the appropriate geometrical factors. Each frame shows the pulsed (open circles) and the DC (full lines) $J-E$ characteristics; the straight dotted lines represent the ohmic values extrapolated from the DC results at very low currents. Figure 1 shows the $J-E$ characteristics of a sample of $\mathrm{La}_{1.2} \mathrm{Sr}_{1.8} \mathrm{Mn}_{2} \mathrm{O}_{7}$, a double layer manganite, below its Curie temperature $\left(T_{\mathrm{C}}\right)$, Fig. 2 - of samples of $\mathrm{CO}$ manganites $\mathrm{Pr}_{2 / 3} \mathrm{Ca}_{1 / 3} \mathrm{MnO}_{3}$ and $\mathrm{Bi}_{1 / 2} \mathrm{Sr}_{1 / 2} \mathrm{MnO}_{3}$, at temperatures below $T_{\mathrm{CO}}$, and Fig. 3 - of samples of two ferrimagnetic double perovskites $\mathrm{Sr}_{2} \mathrm{FeMoO}_{6}$

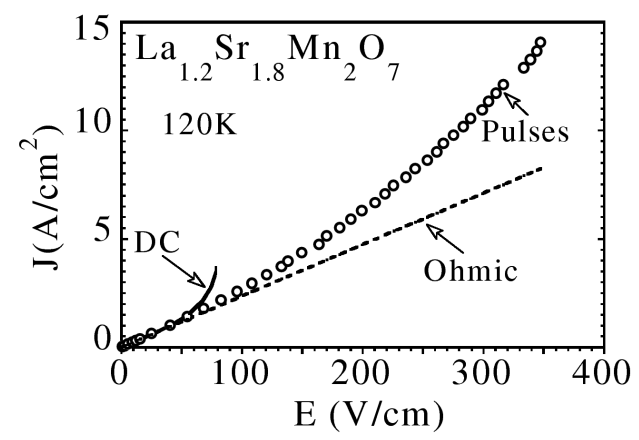

Fig. 1. Pulsed (open circles) and DC (solid line) $J(E)$ for a sample of $\mathrm{La}_{1.2} \mathrm{Sr}_{1.8} \mathrm{Mn}_{2} \mathrm{O}_{7}$, a double layer manganite. The dotted line represents the extrapolated ohmic $J(E)$. 

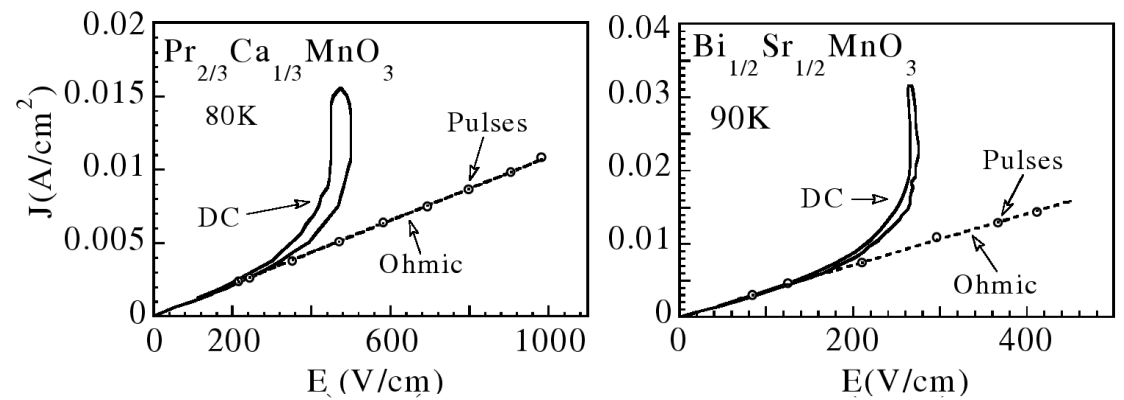

Fig. 2. Pulsed (open circles, dotted lines) and DC (solid lines) $J(E)$ for samples of charge-ordered manganites $\operatorname{Pr}_{2 / 3} \mathrm{Ca}_{1 / 3} \mathrm{MnO}_{3}$ and $\mathrm{Bi}_{1 / 2} \mathrm{Sr}_{1 / 2} \mathrm{MnO}_{3}$. Let us note the perfectly ohmic pulsed $J(E)$.
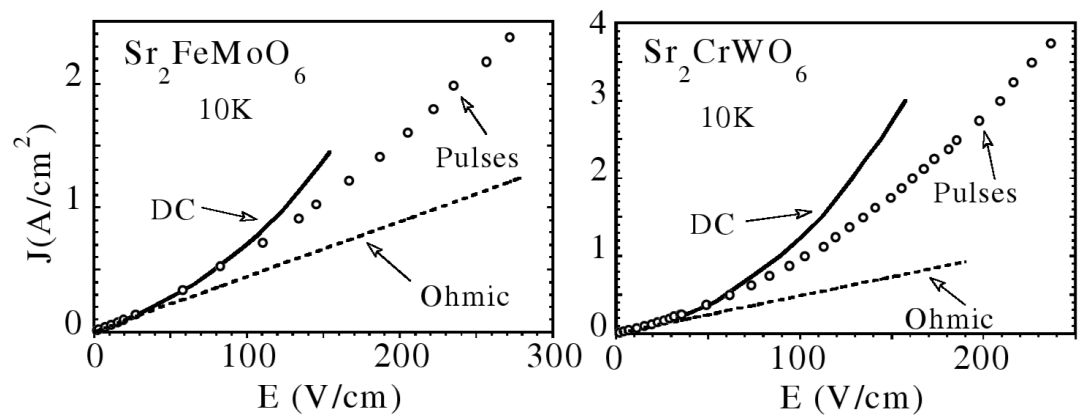

Fig. 3. Pulsed (open circles) and DC (solid lines) $J(E)$ for samples of two double perovskites $\mathrm{Sr}_{2} \mathrm{FeMoO}_{6}$ and $\mathrm{Sr}_{2} \mathrm{CrWO}_{6}$. The dotted lines represent the extrapolated ohmic $J(E)$.

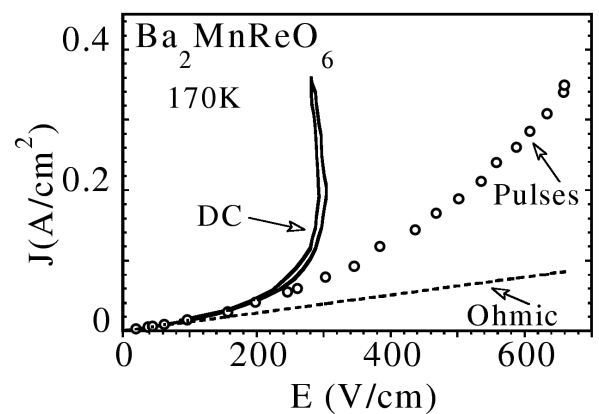

Fig. 4. Pulsed (open circles) and DC (solid line) $J(E)$ for a sample of $\mathrm{Ba}_{2} \mathrm{MnReO}_{6}$. The dotted line represents the extrapolated ohmic $J(E)$.

and $\mathrm{Sr}_{2} \mathrm{CrWO}_{6}$ below their respective $T_{\mathrm{C}}$. Finally, Fig. 4 shows the $J-E$ characteristics of a sample of $\mathrm{Ba}_{2} \mathrm{MnReO}_{6}$, an insulating double perovskite, above its $T_{\mathrm{C}}$. The most remarkable result is shown in Fig. 2, for the well-known CO manganites. The pulsed $J(E)$ plots are linear up to very high electric fields. It should 
be recalled that it was the report on the dramatic behavior of a $\mathrm{Pr}_{2 / 3} \mathrm{Ca}_{1 / 3} \mathrm{MnO}_{3}$ sample under a high electric field that triggered the intense activity in the nonlinear conductivity of $\mathrm{CO}$ manganites [7].

The nonlinearity of the $J(E)$ plots in Figs. 1 and 3, which is consistent also with the temperature dependence of their ohmic conductivity, is associated with inter-grain tunneling $[10,12]$. The nonlinearity of the pulsed $J(E)$ of $\mathrm{Ba}_{2} \mathrm{MnReO}_{6}$ and of other materials from this family is now under investigation in our group. All DC $J(E)$ characteristics shown here are strongly nonlinear; the more extended plots (in Figs. 2 and 4) show ranges of negative resistance and hysteresis. None provide useful information about interesting electronic processes. Nevertheless, reports on similar DC $I-V$ characteristics and related phenomena have filled many pages of important scientific journals.

In conclusion, the DC measurement in the nonlinear regime may hide unsuspected traps; it is about time for the $I-V$ characteristics to be measured more carefully, applying the knowledge accumulated over a century.

\section{References}

[1] For reviews and references see: H. Frohlich, Rep. Prog. Phys. 6, 428 (1939); S. Whitehead, Dielectric Breakdown of Solids, Clarendon, Oxford 1951; W. Franz, in: Encyclopedia of Physics, Ed. S. Flugge, Vol. 17, Springer, Berlin 1956, p. 155; D.M. Kroll, Phys. Rev. B 9, 1669 (1974).

[2] S.R. Ovshinski, Phys. Rev. Lett. 21, 1450 (1968).

[3] See e.g. N.F. Mott, Philos. Mag. 24, 911 (1971) and references therein.

[4] D.F. Weirauch, Appl. Phys. Lett. 16, 72 (1970).

[5] For a review see: C.N.R. Rao, J. Phys. Chem. B 104, 5877 (2000).

[6] Y. Tomioka, A. Asamitsu, H. Kuwahara, Y. Moritomo, Y. Tokura, Phys. Rev. B 53, 1689 (1996) and references therein.

[7] A. Asamitsu, Y. Tomioka, H. Kuwahara, Y. Tokura, Nature 388, 50 (1997).

[8] S. Mercone, R. Frésard, V. Caignaert, Ch. Martin, D. Saurel, C. Simon, G. André, P. Monod, F. Fauth, J. Appl. Phys. 98, 23911 (2005).

[9] H. Song, M. Tokunaga, S. Imamori, Y. Tokunaga, T. Tamegai, cond-mat/0607795 and references therein.

[10] K.B. Chashka, B. Fisher, J. Genossar, L. Patlagan, G.M. Reisner, E. Shimshoni, Phys. Rev. B 63, 64403 (2001); K.B. Chashka, B. Fisher, J. Genossar, L. Patlagan, G.M. Reisner, E. Shimshoni, J.F. Mitchell, Phys. Rev. B 65, 134441 (2002).

[11] B. Fisher, J. Genossar, K.B. Chashka, L. Patlagan, G.M. Reisner, Appl. Phys. Lett. 88, 152103 (2006).

[12] B. Fisher, K.B. Chashka, L. Patlagan, G.M. Reisner, Phys. Rev. B 68, 134420 (2003), ibid. 70, 205109 (2004); Curr. Appl. Phys. 4, 518 (2004); Phys. Rev. B 71, 104428 (2005). 